\title{
Role of gastroscopic biopsy of gastric ulcer margins and healed sites in the diagnosis of early gastric cancer: A clinical controlled study of 513 cases
}

\author{
JING-JING WAN ${ }^{1,2^{*}}$, SU-JUAN FEI ${ }^{3 *}$, SHENG-XIANG LV $^{2 *}$, SHU-TANG HAN ${ }^{1}$, \\ XING-GANG MA ${ }^{2}$, DONG-SHENG XU ${ }^{2}$, HONG-MEI CHEN ${ }^{2}$ and JU ZHANG ${ }^{2}$ \\ ${ }^{1}$ Digestive Endoscopy Center, Affiliated Hospital of Nanjing University of Chinese Medicine, Nanjing, Jiangsu 210023; \\ ${ }^{2}$ Department of Gastroenterology, Huaian Hospital Affiliated to Xuzhou Medical University, Huai'an, Jiangsu 223002; \\ ${ }^{3}$ Department of Gastroenterology, Xuzhou Medical University Affiliated Hospital, Xuzhou, Jiangsu 221004, P.R. China
}

Received June 20, 2016; Accepted May 4, 2017

DOI: $10.3892 / \mathrm{ol} .2018 .9156$

\begin{abstract}
The present case study aimed to evaluate the effect of gastroscopic biopsy of gastric ulcer margins and healed sites in the diagnosis of early gastric cancer. A total of 513 patients who were diagnosed with gastric ulcers using gastroscopy between January 1999 and December 2013 were included in the present study and were divided into either the experimental or the control group. In the control group, samples were only taken from the ulcer margin, whereas in the experimental group samples were taken from the ulcer margin and from the ulcer base. In the experimental group, a routine biopsy of the ulcer margin was performed on first examination, and recheck by gastroscopy was performed from the second week. For ulcers that remained unhealed, a biopsy of the ulcer margin was subsequently conducted; however, for healed or almost healed ulcers, a biopsy of the ulcer base was conducted. The duration of follow-up by gastroscopy ranged between 1 week and 24 months. For the control group, a biopsy of the ulcer margin was conducted using the conventional method. The detection rate of the experimental group was 3.88\% (9/232), with 4 cases of gastric cancer confirmed from examinations of the ulcer base. The detection rate of the control group was $1.07 \%(3 / 281)$, which was significantly decreased compared
\end{abstract}

Correspondence to: Dr Shu-Tang Han, Digestive Endoscopy Center, Affiliated Hospital of Nanjing University of Chinese Medicine, 138 Xianlin Road, Nanjing, Jiangsu 210023, P.R. China

E-mail: shutanghanhst@126.com

Dr Xing-Gang Ma, Department of Gastroenterology, Huaian Hospital Affiliated to Xuzhou Medical University, 62 Huaihai Road, Huai'an, Jiangsu 223002, P.R. China

E-mail: hamxg@sina.com

*Contributed equally

Key words: gastric ulcer, healed sites, gastroscopic biopsy, early gastric cancer, gastroscopy with that of the experimental group $(\mathrm{P}=0.0345)$. Overall, patients who underwent regular follow-up gastroscopy following treatment exhibited a markedly increased detection rate of early gastric cancer, suggesting that early cancer may occur in healed gastric ulcer sites.

\section{Introduction}

Gastric cancer is one of the most common types of malignant tumor, with gastric cancer-associated mortality accounting for $23.03 \%$ of all malignant tumors (1), which is second only to lung cancer and ranks first in digestive tract tumors $(2,3)$.

The early diagnosis of gastric cancer improves the prognosis and the postoperative 5-year survival rate of early gastric cancer (EGC) has been recorded at $90 \%$ (4). EGC refers to cancer with an invasion depth limited to the mucosa or mucosa and submucosa, regardless of metastasis in the lymph nodes (5). The median duration of EGC prior to mortality (i.e., the survival time post-diagnosis) is 44 months (6) and if early cancerous lesions in the stomach are identified during this period, the prognosis of patients may be improved. Therefore, the effects of increasing the detection rate of EGC require study (7).

On the basis of the Paris endoscopic classification (8) and according to the gross morphology of lesions, EGC is divided into three types: Protruded type (0-I), flat type (0-II) and depression type (0-III). According to the morphology, the 0-II type is divided into three subtypes: Superficial protruded type (0-IIA), flat type (0-IIB) and superficial depression type (0-IIC). Type III may be more easily identified by gastroscopy and cancer cells may be determined by performing biopsy of tissues. In the progressive stage, gastric cancer may be observed under a gastroscope and cancer cells may be easily determined by biopsy.

According to the staging method by Shinji et al (9), the ulcer course may be divided into three stages: Active (A), healing $(\mathrm{H})$ and scarring $(\mathrm{S})$. Furthermore, each stage may be divided into two phases to provide the following: A1, A2, H1, $\mathrm{H} 2, \mathrm{~S} 1$ and $\mathrm{S} 2$.

A previous study identified that gastric ulcers are a gastric precancerous disease (10), but were primarily aimed 
at situations where gastric ulcers are difficult to heal. Some researchers believe that endoscopic follow-up of benign gastric ulcers is hypothesized to be of limited value; for instance, Thomopoulos et al (11) demonstrated that during the 6-year follow-up of 731 patients with benign gastric ulcers, no case of gastric cancer was identified. In addition, Bustamante et al (12) revealed that no gastric cancer case was identified in 289 patients with benign gastric ulcers. However, in our 10 years of clinical work, (Ma et al, unpublished data), gastric cancer was occasionally identified. In an experimental group of 232 cases of patients with gastric ulcers, in which 9 patients were diagnosed with cancer, 5 cases of gastric cancers were detected from the edges of ulcers at 1, 14, 18, 19 and 22 months later, respectively. In another 4 cases, gastric cancers were detected from the base of ulcers at 1, 6, 17 and 24 months later, respectively. By repeated gastroscopic biopsy of the healed gastric ulcer site and was diagnosed as EGC by surgery and pathology. Mañas et al (13) identified that 8/452 patients with benign gastric ulcers were diagnosed with gastric cancer (detection rate, 1.8\%) during follow-up. Therefore, we hypothesized that for healed or currently healing chronic gastric ulcers, the biopsy of the ulcer base during gastroscopic follow-up may improve the diagnosis of EGC compared with the traditional method of the biopsy of gastric ulcer margins.

Patients diagnosed with benign gastric ulcer at the Endoscopy Center of the Affiliated Huaian Hospital of Xuzhou Medical University (Huai'an, China), were randomly divided into two groups: An experimental group and a control group. Furthermore, the patients underwent regular gastroscopic rechecks to assess whether the biopsy of the ulcer margins and base may improve the detection rate of EGC compared with the biopsy of ulcer margins alone.

\section{Patients and methods}

Selection of subjects and criteria. The present study was approved by the Ethics Committee of the Affiliated Huaian Hospital of Xuzhou Medical University and all patients provided written informed consent. Between January 1999 and December 2002 14, 202 patients (Fig. 1) underwent gastroscopic examinations in the Endoscopy Center and gastric ulcer patients were rechecked at least twice by gastroscopy. Patients were followed-up for between 1 week and 24 months. A total of 281 patients were selected as controls and these patients underwent biopsy of the ulcer margins only. Following diagnosis with a benign ulcer, patients underwent 8 weeks of regular medical treatment and gastroscopic recheck started from the second week. If the ulcer began to heal, biopsy was no longer conducted. If the ulcer did not heal, only a biopsy of the ulcer margins was conducted. Between January 2006 and December 2009 and between March 2012 and December 2013, 28,142 patients (Fig. 1) underwent gastroscopic examinations in the Endoscopy Center. Gastric ulcer patients were rechecked at least three times by gastroscopy and were followed up for between 1 week and 24 months. A total of 243 patients from this period were included in the experimental group, 11 of whom were lost to follow-up, and so 232 patients completed the study. Patients in the experimental group underwent biopsy of the ulcer margins. When diagnosed with a benign ulcer, patients underwent 8 weeks of regular medical treatment and gastroscopic recheck began from the second week. If the ulcer healed, only biopsy of the ulcer margins was conducted; however, if the ulcer healed or was currently healing, biopsy of the ulcer base was conducted. Clinical, endoscopic and pathological examination data were collected from the two groups of patients. During the last 4 weeks prior to gastroscopic examination, all patients did not use proton pump inhibitors, Bismuth agents, $\mathrm{H}_{2}$ receptor antagonists or antibiotics.

Tissue samples. All 513 selected patients (281 patients in the control group and 232 patients in the experimental group) underwent regular gastroscopic biopsy. Patients in the control group underwent biopsy of the ulcer margins only. Patients in the experimental group underwent a biopsy of the ulcer margins during the active stage of the ulcer, and a biopsy of the ulcer margins and base if the ulcer healed or was currently healing. Between 4 and 6 sections of biopsy samples were obtained using disposable biopsy forceps, and were sent to the Department of Pathology of The Affiliated Huaian Hospital of Xuzhou Medical University for histopathological diagnosis. The gastroscopy and biopsy records of all patients were complete.

Exclusion criteria of disease cases. Patients with the following were excluded: Acute erosive gastritis, cancerous ulcers, compound ulcers and multiple gastric ulcers; patients diagnosed with benign ulcers by visual observation, but diagnosed with gastric cancer during the initial biopsy; and patients with gastric ulcers occurring after gastric cancer surgery.

Research methods. The epidemiological features of patients included the following: Sex, age, smoking history and the administration of non-steroidal anti-inflammatory drugs (NSAIDs).

Investigation method of the administration history of NSAIDs. Regardless of dose and duration, the oral administration of NSAIDs prior to gastroscopic examination was defined as the administration of NSAIDs.

Helicobacter pylori detection using rapid urease. The white square silicone oil slip sheet of the $H$. pylori test paper was removed and biopsy samples were placed in the center of the round yellow paper. Subsequently, the adhesive paper was fixed on the plastic in order to fix the test paper with biopsy tissues to the plastic. The results were observed under normal temperature conditions. The alteration in the color of the margins of the biopsy tissue on the test paper determined the judgment criteria, as follows: Strong positive result, yellow to red within $1 \mathrm{~min}$; weak positive result, yellow to red within $3 \mathrm{~min}$; and negative result, no change. The outcome was invalid if a change occurred after $3 \mathrm{~min}$.

Helicobacter pylori detection by ${ }^{13} \mathrm{C}$ expiration. The investigation was performed on patients either in the morning, when stomach was empty, or following $>2 \mathrm{~h}$ fasting. Patients filled out the required information on the two labeled air bags and subsequently blew air into the bags in a normal manner until the bags were full. The bag covers were then immediately tightened. The air collected was defined as 


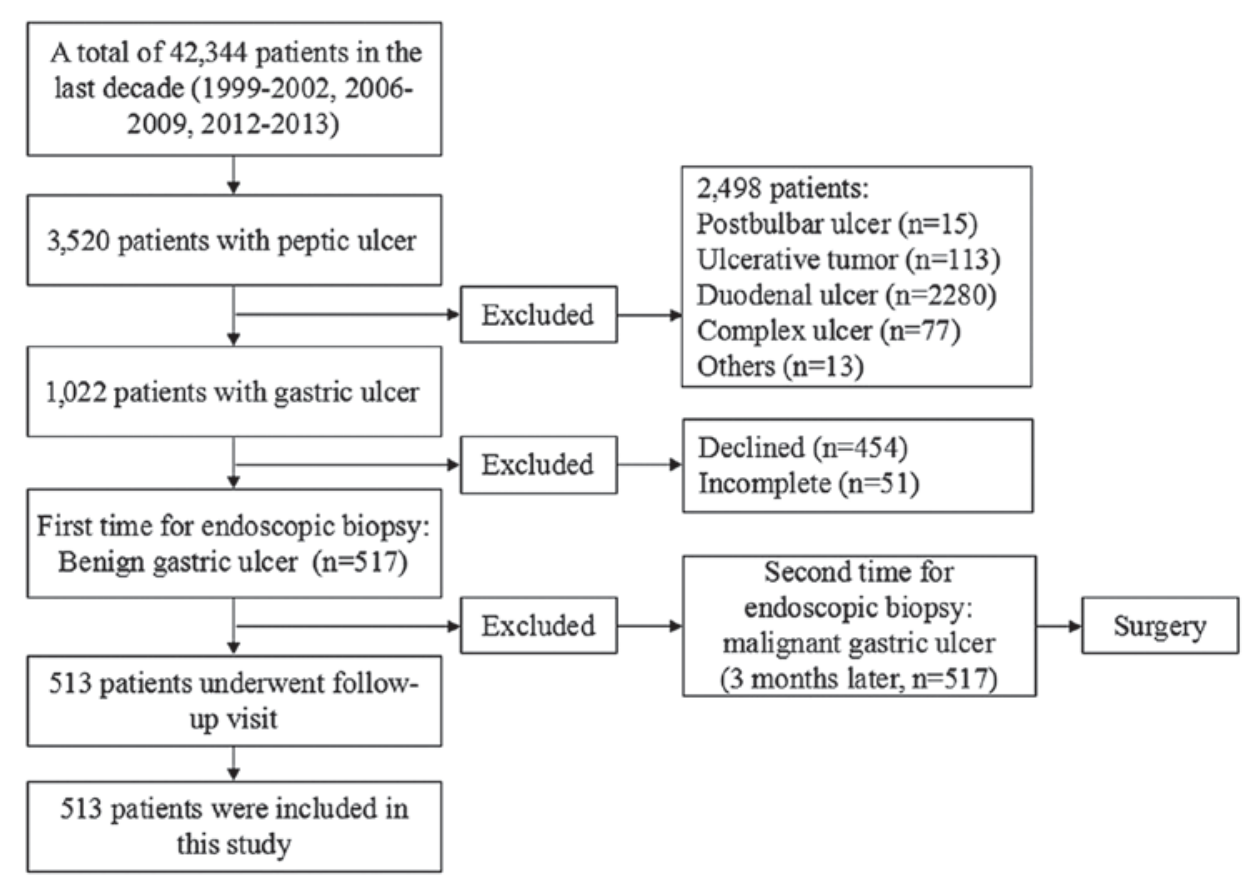

Figure 1. Flowchart explaining the process of the inclusion and exclusion of patients in the present study.

the ' 0 -min expiration air'. The subjects took one urea ${ }^{13} \mathrm{C}$ capsule with between 80 and $100 \mathrm{ml}$ cold drinking water and sat quietly. After $30 \mathrm{~min}$, expiration air was collected from the subjects as aforementioned, and the air collected was defined as the ' 30 -min expiration air'. '0-min expiration air' and '30-min expiration air' were used for ${ }^{13} \mathrm{CO}_{2}$ detection, with $\delta \%$ (parts/thousand) used to represent the results. The following formula was used: $\delta \%_{0}=\left({ }^{13} \mathrm{C}\right.$ abundance ${ }_{\text {determined }}$ sample ${ }^{-13} \mathrm{C}$ abundance ${ }_{\text {reference sample }}$ ) $\mathrm{x} 1000 /{ }^{13} \mathrm{C}$ abundance ${ }_{\text {reference }}$ sample. The diagnosis of $H$.pylori was determined on the basis of the difference between the $\delta \%$ values of the ' 0 -min expiration air' minus and that of the '30-min expiration air' (detection value $=\delta \%$ at $30 \mathrm{~min}-\delta \%$ at $0 \mathrm{~min}$ ). When the positive judgment value was $\geq 4.0 \pm 0.4$ (mean \pm standard deviation), subjects were defined as $H$. pylori-positive.

Judgment criteria of $H$. pylori infection. When the urease and ${ }^{13} \mathrm{C}$ expiration tests presented positive results, or when urease was negative and ${ }^{13} \mathrm{C}$ was positive, the patient was identified as infected with $\mathrm{H}$. pylori. If the urease and ${ }^{13} \mathrm{C}$ expiration tests presented negative results, or if the urease test was positive and ${ }^{13} \mathrm{C}$ expiration test was negative, the patient was identified to be negative for $H$. pylori.

Medical treatment scheme for gastric ulcer. Treatment for $H$. pylori-positive gastric ulcers was as follows: Rabeprazole sodium enteric-coated capsules, $20 \mathrm{mg}$, twice/day for 14 days; colloidal bismuth pectin dry suspension, $150 \mathrm{mg}$, three times/day for 14 days; clarithromycin sustained-release tablets, $500 \mathrm{mg}$, twice/day for 14 days; and amoxicillin, 1,000 mg, twice/day for 14 days. After 14 days of treatment, clarithromycin sustained-release tablets and amoxicillin were ceased, and the administration of rabeprazole sodium enteric-coated capsules and colloidal bismuth pectin dry suspension was continued for 6 weeks. Treatment for $H$. pylori-negative gastric ulcers was as follows: Rabeprazole sodium enteric-coated capsules, $20 \mathrm{mg}$ per day for 8 weeks; and colloidal bismuth pectin dry suspension, $150 \mathrm{mg}$, three times/day for 8 weeks

Gastroscopy. All patients were examined using the Olympus GIF-XQ240 and GI-XQ260 series electronic gastroscope (Olympus Corporation, Tokyo, Japan) and all examinations were completed by two experienced endoscopic physicians.

Preparation prior to the examination. All patients were examined with an empty stomach in the early morning. A total of $10 \mathrm{mg}$ racanisodamine hydrochloride was intramuscularly injected $5 \mathrm{~min}$ prior to the examination. Subsequently, 1 bottle of dyclonine hydrochloride mucilage was orally taken $(0.1 \mathrm{~g}, 10 \mathrm{ml})$.

Examination steps. A conventional endoscope was inserted and reached the descending part of the duodenum, and the pyloric canal, gastric antrum, gastric body and gastric fundus were observed during the withdrawal of the gastroscope. If suspected lesions were identified, lesion morphology, margins and color distinctions of the surrounding area were preliminarily observed and images were acquired. The majority of benign ulcers were round or elliptical, although certain ulcers were linear with smooth margins. The bottom was covered with yellowish gray or grayish white exudate, the surrounding mucosa presented with congestion and edema, and the plicae around the ulcer were centrally located. Biopsy tissues were obtained from a number of lesion sites using disposable biopsy forceps for pathological examination.

Sampling. Patients in the control group underwent biopsy of the ulcer margins only. When diagnosed with a benign ulcer, patients underwent 8 weeks of regular medical treatment with gastroscopic recheck beginning from the second week. If the 


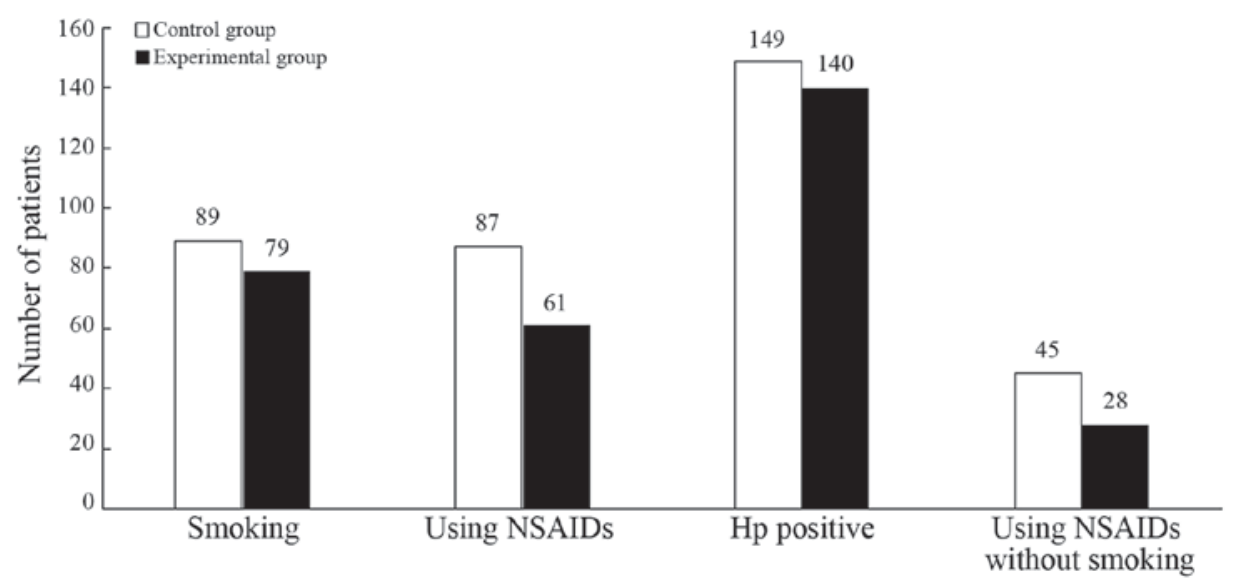

Figure 2. Smoking, NSAID and Helicobacter pylori history of patients in control and experimental groups. NSAID, non-steroidal anti-inflammatory drugs; Hp, Helicobacter pylori.

ulcer began to heal, biopsy was no longer conducted; however, if the ulcer did not heal, biopsy of the ulcer margins was conducted. The duration of follow-up by gastroscopy ranged between 1 week and 24 months. Patients in the experimental group underwent biopsy of the ulcer margins alone in the initial gastroscopy, and following diagnosis with a benign ulcer, the patients underwent 8 weeks of regular medical treatment, with gastroscopic recheck beginning from the second week. During the healing process of the ulcer, the ulcer margins were prioritized as the site of gastroscopic biopsy. If the ulcers healed or were currently healing, biopsy samples were obtained from the ulcer base or the healed sites. Gastroscopy rechecks in the experimental group were conducted on weeks 2, 4 and 8, and thereafter once a year. When gastric cancer was identified, patients were surgically treated, followed by gross histopathological examination. From each ulcer lesion, between 4 and 6 biopsy samples were obtained.

Pathological examination. All biopsy samples were placed on filter paper, fixed in $10 \%$ formalin solution and sent to the Department of Pathology of The Affiliated Huaian Hospital of Xuzhou Medical University (Huai'an, China) for histopathological diagnosis. All biopsies were conducted by experienced pathologists following fixation in $10 \%$ formalin solution, then dehydration, clearing, and infiltrating were finished in the machine overnight $(12 \mathrm{~h})$, embedding in paraffin $\left(\sim 2 \mathrm{~h}\right.$ at $\left.58^{\circ} \mathrm{C}\right)$. Sections were sliced at a thickness of $\sim 4 \mu \mathrm{m}$. Subsequently, sections were subjected to hematoxylin eosin staining: i) The sections were deparaffinized, flamed using a burner and then placed in the $>99 \%$ xylene. This step was repeated 2 times; ii) the tissues were hydrated by passing through decreasing concentrations of alcohol and water $(100,90,80$ and $70 \%)$; iii) the sections were stained in hematoxylin $(1 \mathrm{~g})$ for $5 \mathrm{~min}$ at room temperature; iv) and were then washed with tap water for $\leq 5$ min, until a blue color change was observed; v) excess dye was removed and the clear staining effect achieved following the addition of $1 \%$ acid alcohol (1\% HCL in $70 \%$ alcohol) for $5 \mathrm{~min}$; vi) and were then washed in running tap water until the sections were again blue by dipping in an alkaline solution followed by another tap water wash; vii) the sections were stained with $1 \%$ eosin for $10 \mathrm{~min}$; viii) and were then washed in tap water for $5 \mathrm{~min}$; ix) the sections were dehydrated in increasing concentrations $(85,90,95,10$ and 100\%) of alcohols and placed in $>99 \%$ xylene; and $\mathrm{x}$ ) a neutral balsam neutral gum (Sinopharm Chemical Reagent Co., Ltd., Shanghai, China) was used to seal the sections.

Statistical analysis. Data were recorded using Windows Excel (2007; Microsoft Corporation, Redmond, WA, USA) and analyzed using Stata for Windows (version 12.0; StataCorp LP, College Station, TX, USA). Data are expressed as the mean \pm standard deviation and count data were expressed as percentages. Continuous variables, including age, were compared using Student's t-test and discrete variables were compared using the $\chi^{2}$ test. The inspection level was $\alpha=0.05$ and $\mathrm{P}<0.05$ was considered to indicate a statistically significant difference.

\section{Results}

Epidemiological characteristics of patients in the two groups. Sex and age. In the control group, 185/281 patients were male and $96 / 281$ patients were female, with a male-to-female ratio of 1.93:1. The age of patients in the control group ranged between 30 and 80 years, with a mean age of $53.9 \pm 12.7$ years. In the experimental group, $139 / 232$ patients were male and 93/232 patients were female, with a male-to-female ratio of 1.49:1. The age of patients in the experimental group ranged between 30 and 83 years, with a mean age of $55.8 \pm 12.0$ years.

Previous medical history. In the control group, 89 patients (31.7\%) exhibited a history of smoking, 87 patients $(31.0 \%)$ possessed a history of NSAID administration, 149 patients (53.0\%) had a H. pylori-positive history, and 45 patients $(16.0 \%)$ had never smoked and never used NSAIDs. In the experimental group, 79 patients $(34.1 \%)$ exhibited a history of smoking, 61 patients $(26.3 \%)$ possessed a history of NSAID administration, 140 patients $(60.3 \%)$ have a $H$. pylori-positive history, and 28 patients (12.1\%) have never smoked and never used NSAIDs (Fig. 2).

Statistical analysis of epidemiological characteristics. Differences between the epidemiological characteristics of the 
Table I. Statistical analysis of epidemiological features between the experimental group and the control group.

\begin{tabular}{|c|c|c|c|}
\hline Feature & Experimental group $(n=232)$ & Control group $(n=281)$ & P-value \\
\hline \multicolumn{4}{|l|}{ Sex, $n$} \\
\hline Male & 139 & 185 & \multirow[t]{2}{*}{$0.167^{\mathrm{a}}$} \\
\hline Female & 93 & 96 & \\
\hline Mean age, years & $55.8 \pm 12.0^{\mathrm{b}}$ & $53.9 \pm 12.7$ & $0.080^{\mathrm{c}}$ \\
\hline \multicolumn{4}{|c|}{ Smoking history, $\mathrm{n}$} \\
\hline Smoking & 79 & 89 & \multirow[t]{2}{*}{$0.568^{\mathrm{a}}$} \\
\hline No smoking & 153 & 192 & \\
\hline \multicolumn{4}{|l|}{ NSAID use, $\mathrm{n}$} \\
\hline Yes & 61 & 87 & \multirow[t]{2}{*}{$0.245^{\mathrm{a}}$} \\
\hline No & 171 & 194 & \\
\hline \multicolumn{4}{|l|}{ Hp infection, $\mathrm{n}$} \\
\hline Yes & 140 & 149 & \multirow[t]{2}{*}{$0.096^{\mathrm{a}}$} \\
\hline No & 92 & 132 & \\
\hline \multicolumn{4}{|c|}{ NSAID use without smoking, $\mathrm{n}$} \\
\hline Yes & 28 & 45 & \multirow[t]{2}{*}{$0.201^{\mathrm{a}}$} \\
\hline No & 204 & 236 & \\
\hline
\end{tabular}

${ }^{\mathrm{a}} \chi^{2}$ test; ${ }^{\mathrm{b}}$ mean \pm standard deviation; 'Student's t-test. NSAID, non-steroidal anti-inflammatory drugs; Hp, Helicobacter pylori.

Table II. Clinical features of the 9 patients diagnosed with gastric cancer in the experimental group.

\begin{tabular}{lccccccccc}
\hline & \multicolumn{1}{c}{ Patient no. } \\
\cline { 2 - 10 } Feature & 1 & 2 & 3 & 4 & 5 & 6 & 7 & 8 \\
\hline Sex & M & M & F & M & F & F & M & F & M \\
Age, years & 65 & 57 & 48 & 70 & 63 & 49 & 56 & 67 & 55 \\
Hp infection & Yes & Yes & Yes & Yes & Yes & Yes & Yes & Yes & Yes \\
History of NSAIDs use & No & No & No & Yes & No & Yes & No & Yes & No \\
Biopsy blocks, n & 5 & 4 & 6 & 5 & 4 & 4 & 6 & 7 & 5 \\
Gastric cancer location in ulcer & Base & Margin & Base & Margin & Margin & Base & Margin & Margin & Base \\
\hline
\end{tabular}

M, male; F, female; Hp, Helicobacter pylori; NSAIDs, non-steroidal anti-inflammatory drugs.

two groups of patients were not identified to be statistically significant $(\mathrm{P}>0.05$; Table I).

Gastric cancer cases determined in patients. In the experimental and control groups overall, $12(2.34 \%)$ patients with a gastric ulcer were diagnosed with gastric cancer. Furthermore, gastric cancer was identified in 9 patients in the experimental group (detection rate, $3.88 \%$ ), with 5 of these patients diagnosed with gastric cancer at 1, 14, 18, 19 and 22 months, respectively, following the diagnosis of gastric ulcer. Diagnoses were validated by biopsies obtained from the margins of the ulcer that had healed, or almost healed. The remaining 4 patients were diagnosed with gastric cancer at 1, 6, 17 and 24 months, respectively, following the diagnosis of the gastric ulcer, which was subsequently confirmed by biopsy of the base of the healed ulcers (Table II). All 9 patients with gastric cancer underwent surgical treatment and were validated as patients with EGC by postoperative pathology. In the control group, 3/281 patients were diagnosed with gastric cancer (detection rate, $1.07 \%$ ) at 3, 15 and 16 months, respectively, following the diagnosis of gastric ulcer, and were validated by biopsy of the margins of the almost healed ulcers (Table III). All 3 patients with gastric cancer underwent surgical treatment and were diagnosed with EGC by postoperative pathology.

Statistical comparison of EGC cancer detection rate between the two groups. The detection rate of gastric cancer in patients in the experimental group and control group was 3.88 and $1.07 \%$, respectively. The difference was identified to be statistically significant $(\mathrm{P}<0.05$; Table IV).

Comparison of $H$. pylori infection rate between patients with EGC and non-gastric cancer patients. A total of 12/513 patients with gastric ulcers in the two groups were diagnosed 
with EGC, with all 12 patients identified to be infected with H. pylori (infection rate, 100\%). Among the gastric ulcer patients who were not diagnosed with gastric cancer, 277/501 patients were diagnosed with $H$. pylori infection (infection rate, $55.29 \%)$. The difference between the two groups was identified to be statistically significant $(\mathrm{P}<0.05$; Table $\mathrm{V})$.

\section{Discussion}

With the decreasing incidence of gastric cancer and the wide use of NSAIDs following identification of benign gastric ulcers by gastroscopic observation and biopsy pathology, the necessity of regular follow-ups by gastroscopy remains controversial in Western countries (13-15). A previous study revealed that in 144 patients with a benign gastric ulcer, $7 \%$ patients were eventually diagnosed with gastric cancer (16). Todd et al (17) hypothesized that, if ulcers were diagnosed to be benign by endoscopic observation and were pathologically confirmed, all patients with gastric ulcer require follow-up by gastroscopy in order to assess ulcer healing and the occurrence of malignant transformation. Furthermore, the British Society of Gastroenterology guidelines (18) emphasize the value of follow-up by gastroscopy for patients with gastric ulcers. Eckardt et al (19) identified that $8 / 452$ patients with benign gastric ulcers were diagnosed with gastric cancer (detection rate, 1.8\%) during follow-up and demonstrated that regular follow-up by gastroscopy may improve the detection rate of EGC. Hopper et al (20) identified that follow-up by gastroscopy may enable earlier detection of gastric cancer, improving the survival time of patients with gastric cancer. In addition, Podolsky et al (21) revealed that 6 patients with gastric ulcers were diagnosed with gastric cancer during follow-up by gastroscopy and that 2 of these patients exhibited EGC, with lesions limited to the mucosa and submucosa and no lymph node metastasis. Furthermore, a previous study revealed that, despite the use of typical treatments (including the eradication of H.pylori), a number of patients with benign gastric ulcer exhibited disease progression and developed an ulcer with an undetermined nature, A limited number of these patients may have a gastric cancer type, including lymphoma (22).

It has been previously demonstrated that the likelihood of determining whether a benign ulcer will exhibit canceration during follow-up of patients is low; however, the cost-benefit ratio is high $(11,12,14)$. Furthermore, a previous study identified patients to exhibit ulcer canceration during the follow-up, but their prognosis did not improve (19). In addition, a previous study revealed that it is inadequate to diagnose the nature of a disease only by visual inspection under an endoscope; therefore, biopsy is required (23). In previous studies, the detection rate of gastric cancer in gastric ulcer patients, according to gastroscopy and pathology during follow-up, was between 1.3 and $13.3 \%(16,24)$. Therefore, it is necessary to perform regular gastroscopy on gastric ulcer patients in order to detect early canceration. A previous study revealed that gastric ulcers may heal as a result of effective anti-ulcer drug treatment (5). In addition, sampling from the base of the healed ulcer for biopsy may assist in detecting gastric cancer at an earlier stage (25). Banerjee et al (26) hypothesized that a biopsy was necessary after the ulcer healed, that samples
Table III. Clinical features of 3 patients diagnosed with gastric cancer in the control group.

\begin{tabular}{lccc}
\hline & \multicolumn{3}{c}{ Patient no. } \\
\cline { 2 - 4 } Feature & 1 & 2 & 3 \\
\hline Sex & M & F & M \\
Age, year & 63 & 55 & 61 \\
Hp infection & Yes & Yes & Yes \\
History of NSAIDs use & No & No & Yes \\
Biopsy blocks, $n$ & 5 & 4 & 6 \\
Gastric cancer location in ulcer & Margin & Margin & Margin
\end{tabular}

M, male; F, female; Hp, Helicobacter pylori; NSAIDs, non-steroidal anti-inflammatory drugs.

may be obtained from the base of the ulcer, that patients may be followed up for $>1$ year and that it was incorrect to consider a healed ulcer as a benign lesion. Additionally, Esmadi et al (27) hypothesized that gastric ulcers may be cured and demonstrated that patients should undergo regular gastroscopic recheck. Podolsky et al (21) identified that a biopsy of the healed sites of the gastric ulcer was required. However, to date, there have been no large-sample studies in which, following healing of a gastric ulcer, EGC has been determined using gastroscopy, pathological follow-up examinations and biopsies of the healed site.

In the present study, 513 patients with gastric ulcers were followed up for between 1 week and 24 months. In the experimental group, 9/232 patients developed EGC and $H$. pylori infection, with a gastric cancer detection rate of $3.88 \%$, which was consistent with the results found by Ogura et al (28). Furthermore, after the ulcers had almost or completely healed, 9 cases of gastric cancer were determined by biopsy of the margins or base of the ulcer, and the detection rate of EGC was increased compared with that of the control group. The results of the present study suggested that the additional biopsy of the ulcer base during the healing process may improve the detection rate of EGC.

In the present study, the H.pylori infection rate was $56.3 \%$ and patients with EGC exhibited a significantly increased $H$. pylori infection rate compared with patients with gastric ulcers alone, suggesting that $H$. pylori infection is associated with gastric cancer. The results of the present study suggest that, under the same conditions of $H$. pylori infection, atrophic gastritis, intestinal metaplasia and atypical hyperplasia, gastric cancer may develop not only on the ulcer margins, but also on the ulcer base.

An endoscope with advanced auxiliary functions, including magnifying endoscopy, narrow-band imaging endoscopy, staining endoscopy, ultrasound endoscopy and confocal endomicroscopy, would enable the local mucosal structure and layer structures of tissues to be observed. Additionally, experience of gastroscopy technology and the endoscopic biopsy technique continues to increase, which may improve the detection rate of EGC. In the present study, the biopsy of the ulcer base is hypothesized to be of increased importance 
Table IV. Comparison of gastric cancer detection rate between the control and experimental groups.

\begin{tabular}{lcccc}
\hline Group & $\begin{array}{c}\text { No. of positive } \\
\text { gastric cancer cases }\end{array}$ & $\begin{array}{c}\text { No. of negative } \\
\text { gastric cancer cases }\end{array}$ & Total & $\begin{array}{c}\text { Detection } \\
\text { rate, \% }\end{array}$ \\
\hline Experimental & 9 & 223 & 232 & 3.88 \\
Control & 3 & 278 & 281 & 1.07 \\
\hline
\end{tabular}

${ }^{\mathrm{a}} \chi^{2}$ test; $\chi^{2}=4.502$

Table V. Comparison of the Helicobacter pylori infection rate between patients with early gastric cancer and patients without gastric cancer.

\begin{tabular}{lcccc}
\hline Patients & No. of Hp positive cases & No. of Hp negative cases & Total & Hp-positive rate, $\%_{\text {P-value }}$ \\
\hline Patients with EGC & 12 & 0 & 12 & 100.0 \\
Non-gastric cancer patients & 277 & 224 & 501 & 55.29 \\
\hline
\end{tabular}

$\chi^{2}=9.524 .{ }^{a} \chi^{2}$ test; ECG, early gastric cancer; Hp, Helicobacter pylori.

since it may be more effective and enable the detection of gastric cancer at an earlier stage. To the best of our knowledge, there are no studies on its underlying molecular mechanism of occurrence on a global scale. It is hypothesized that a gastric ulcer may occur on the basis of EGC. If the malignant degree of cancer cells is decreased, the invasion and damage effects of cancer cells are less than the proliferation effect of the cancerous tissue itself, however, if cancerous tissues are submucosal infiltrating growth, the ulcer may shrink and even completely heal. Therefore, early cancer lesions may be identified in healed gastric ulcers, which may explain clinical ulcer repair in type II and III gastric ulcers, but does not explain the occurrence of EGC in healed ulcer sites. However, the occurrence of early cancer lesions in the gastric ulcer healed sites may be due to the mutation of submucosal cells in the ulcer base. During the process of ulcer repair, cancer cells (EGC) gradually transition to the mucosal surface where, over time and following alterations in the internal and external environment, these cells progress to advanced gastric cancer. This hypothesis may explain EGC that is identified following the repair of large gastric ulcers.

In the experimental and control groups, 9/232 and 3/281 patients were diagnosed with EGC, respectively (detection rates, 3.88 and $1.07 \%$, respectively), and the difference between the two groups was identified to be statistically significant. The results of the present study suggested that gastroscopy and pathological follow-up of chronic gastric ulcers, during and after the healing process, is required and is of greater importance in regions with an increased incidence of gastric cancer. In addition, biopsy of the base or margins of healing or healed ulcers in gastroscopic follow-ups is important. At present, in China, patients with chronic gastric ulcer may not fully understand the seriousness of ulcerated lesions. Clinicians may not realize the importance of gastroscopic biopsy and the biopsy site of healing or healed gastric ulcers. Therefore, additional studies on this topic and large-scale multicenter randomized controlled trials of chronic gastric ulcers are required.

\section{Acknowledgements}

Not applicable.

\section{Funding}

The present study was supported by the Science and Technology Bureau of Huai'an city (grant no. has2010016).

\section{Availability of data and materials}

The datasets generated and analyzed in the present study are included in this published article.

\section{Authors' contributions}

$\mathrm{SH}, \mathrm{XM}$ and JW were responsible to study design and method selection. JW, SL and DX performed gastroscopy. JW and SF performed data collection, and JW and SF performed data arrangement. JW, SF, SH and XM performed statistical analysis. JW, SH and XM reviewed and revised the manuscript. $\mathrm{HC}$ and $\mathrm{JZ}$ were responsible for the registration of the patients and the collection of the specimens.

\section{Ethics approval and consent to participate}

The present study was approved by The Ethics Committee of the Affiliated Huaian Hospital of Xuzhou Medical University. All patients provided written informed consent to participate.

\section{Consent for publication}

All patients consented to the publication of this research.

\section{Competing interests}

The authors declare that they have no competing interests. 


\section{References}

1. Birner P, Schindl M, Obermair A, Plank C, Breitenecker G and Oberhuber G: Over expression of hypoxia-inducible factor lalpha is a marker for an unfavorable prognosis in early-stage invasive cervical cancer. Cancer Res 60: 4693-4696, 2000.

2. Forman D and Burley VJ: Gastric cancer: Global pattern of the disease and an overview of environmental risk factors. Best Prac Res Clin Gastroenterol 20: 633-649, 2006.

3. Brenner H, Rothenbacher D and Arndt V: Epidemiology of stomach cancer. Methods Mol Biol 472: 467-477, 2009.

4. Dong ZW: The guidelines for the screening and early diagnosis and treatment of cancer in China. Peking Univ Med Press 1-77, 2005.

5. Xu SF and Zhao ZQ: Current therapy evolved of early gastric cancer. Med J CASC 3: 74-76, 2001.

6. Tsukuma H, Oshima A, Narahara H and Morii T: Natural history of early gastric cancer: A non-concurrent, long term, follow up study. Gut 47: 618-621, 2000.

7. Gadducci A, Ferdeghini M, Prontera C, Moretti L, Mariani G, Bianchi R and Fioretti P: The concomitant determination of different tumor markers in patients with epithelial ovarian cancer and benign ovarian masses: Relevance for differential diagnosis. Gynecol Oncol 44: 147-154, 1992.

8. The Paris Endoscopic Classification of Superficial Neoplastic Lesions: Esophagus, stomach, and colon: November 30 to December 1, 2002. Gastrointest Endosc 58(6 Suppl): S3-S43, 2003.

9. Shinji A,SanoK,HamanoH,UnnoH,FukushimaM,NakamuraN, Akamatsu T, Kawa S and Kiyosawa K: Autoimmune pancreatitis is closely associated with gastric ulcer presenting with abundant IgG4-bearing plasma cell infiltration. Gastrointest Endosc 59: 506-511, 2004 .

10. Rugge M, Capelle LG, Cappellesso R, Nitti D and Kuipers EJ: Precancerous lesions in the stomach: From biology to clinical patient management. Best Pract Res Clin Gastroenterol 27: 205-223, 2013

11. Thomopoulos KC,Melachrinou MP,Mimidis KP,Katsakoulis EC, Margaritis VG, Vagianos CE and Nikolopoulou VN: Gastric ulcers and risk for cancer. Is follow-up necessary for all gastric ulcers? Int J Clin Pract 58: 675-677, 2004.

12. Bustamante M, Devesa F, Borghol A, Ortuño J and Ferrando MJ: Accuracy of the initial endoscopic diagnosis in the discrimination of gastric ulcers: Is endoscopic follow-up study always needed? J Clin Gastroenterol 35: 25-28, 2002.

13. Mañas MD, Domper A, Albillos A, Hernández A, Carpintero $P$ Lorente R, López B, De la Santa E, Olmedo J and Rodríguez E: Endoscopic follow-up of gastric ulcer in a population at intermediate risk for gastric cancer. Rev Esp Enferm Dig 101: 317-324, 2009 (Article in English, Spanish).

14. Gielisse EA and Kuyvenhoven JP: Follow-up endoscopy for benign-appearing gastric ulcers has no additive value in detecting malignancy: It is time to individualise surveillance endoscopy. Gastric Cancer 18: 803-809, 2015.

15. Lübbers H, Mahlke R, Lankisch PG and Stolte M: Follow-up endoscopy in gastroenterology: When is it helpful. Dtsch Arztebl Int 107: 30-39, 2010.

16. Farinati F, Cardin F, Di Mario F, Vianello F, Battaglia G, Arslan-Pagnini C, Cannizzaro R, Sava GA, Rugge M and Naccarato R: Early and advanced gastric cancer during follow-up of apparently benign gastric ulcer: Significance of the presence of epithelial dysplasia. J Surg Oncol 36: 263-267, 1987.
17. Todd JA, Richards CJ, Dixon A and Robinson RJ: Gastric ulcer and malignancy-is there a need for follow-up endoscopy? Aliment Pharmacol Ther 19: 989-991, 2004.

18. Allum WH, Blazeby JM, Griffin SM, Cunningham D, Jankowski JA and Wong R; Association of Upper Gastrointestinal Surgeons of Great Britain and Ireland, the British Society of Gastroenterology and the British Association of Surgical Oncology: Guidelines for the management of oesophageal and gastric cancer. Gut 60: 1449-1472, 2011.

19. Eckardt VF, Giessler W, Kanzler G and Bernhard G: Does endoscopic follow-up improve the outcome of patients with benign gastric ulcers and gastric cancer? Cancer 69: 301-305, 1992.

20. Hopper AN, Stephens MR, Lewis WG, Blackshaw GR, Morgan MA, Thompson I and Allison MC: Relative value of repeat gastric ulcer surveillance gastroscopy in diagnosing gastric cancer. Gastric Cancer 9: 217-222, 2006.

21. Podolsky I, Storms PR, Richardson CT, Peterson WL and Fordtran JS: Gastric adenocarcinoma masquerading endoscopically as benign gastric ulcer. A five-year experience. Dig Dis Sci 33: 1057-1063, 1988

22. Cherian PT, Cherian S and Singh P: Long-term follow-up of patients with gastric outlet obstruction related to peptic ulcer disease treated with endoscopic balloon dilatation and drug therapy. Gastrointest Endosc 66: 491-497, 2007.

23. Graham DY, Schwartz JT, Cain GD and Gyorkey F: Prospective evaluation of biopsy number in the diagnosis of esophageal and gastric carcinoma. Gastroenterology 82: 228-231, 1982.

24. Lupano F and Sategna-Guidetti C: Endoscopic follow-up of patients with gastric ulcer. A prospective study. J Clin Gastroenterol 8: 430-434, 1986.

25. Lv SX, Gan JH, Ma XG, Wang CC, Chen HM, Luo EP, Huang XP, Wu SH, Qin AL, Ke-Chen, Wang XH, et al: Biopsy from the base and edge of gastric ulcer healing or complete healing may lead to detection of gastric cancer earlier: An 8 years endoscopic follow-up study. Hepatogastroenterology 59: 947-950, 2012.

26. ASGE Standards of Practice Committee; Banerjee S, Cash BD Dominitz JA, Baron TH, Anderson MA, Ben-Menachem T, Fisher L, Fukami N, Harrison ME, et al: The role of endoscopy in the management of patients with peptic ulcer disease. Gastrointest Endosc 71: 663-668, 2010.

27. Esmadi M, Ahmad DS and Hammad HT: Endoscopic surveillance for gastric ulcers. South Med J 107: 289-291, 2014.

28. Ogura M, Yamaji Y, Hikiba Y, Maeda S, Matsumura M, Okano K, Sassa R, Yoshida H, Kawabe T and Omata M: Gastric cancer among peptic ulcer patients: Retrospective, long-term follow-up. Dig Liver Dis 38: 811-814, 2006.

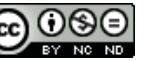

This work is licensed under a Creative Commons Attribution-NonCommercial-NoDerivatives 4.0 International (CC BY-NC-ND 4.0) License. 